\title{
X-RAY MAGNETO-OPTIC KERR EFFECT STUDIES OF SPRING MAGNET HETEROSTRUCTURES
}

\author{
J. B. Kortright, ${ }^{1}$ Sang-Koog Kim, ${ }^{1, *}$ E. E. Fullerton, ${ }^{2}$ J. S. Jiang, ${ }^{3}$ and S. D. Bader ${ }^{3}$ \\ ${ }^{1}$ Materials Sciences Division, Lawrence Berkeley National Lab, Berkeley, California 94720 USA \\ ${ }^{2}$ IBM Almaden Research Center, 650 Harry Road, San Jose, California 95120 USA \\ ${ }^{3}$ Materials Sciences Division, Argonne National Laboratory, Argonne, Illinois
}

\begin{abstract}
The complex 3-dimensional magnetization reversal behavior of $\mathrm{Sm}-\mathrm{Co} / \mathrm{Fe}$ exchange spring films is used to test the sensitivity of different resonant soft x-ray magneto-optical Kerr effect (MOKE) measurements to changes in longitudinal and transverse moments within the soft Fe layer and to changes in these moments in depth within the Fe layer. As in the visible MOKE, changes in longitudinal and net transverse moments are resolved by measuring both Kerr rotation and intensity loops, respectively, near the Fe $2 p$ core resonance. These $\mathrm{x}$-ray MOKE signals measured using linear incident polarization are more directly interpreted in terms of longitudinal and transverse moments than are the same signals measured using elliptical polarization. Varying photon energy near the Fe $L_{3}$ line is shown to be an effective means of resolving distinctly different reversal behavior at the top and bottom of the $20 \mathrm{~nm}$ thick Fe layer resulting from the strong exchange coupling at the $\mathrm{Sm}-\mathrm{Co} / \mathrm{Fe}$ interface. Measured x-ray MOKE spectra and signals are in qualitative agreement with those calculated using standard magneto-optical formalisms incorporating interference between different layers and measured helicity-dependent magneto-optical constants for Fe.
\end{abstract}

PACS: 75.70.-i, 75.75.Cn, 78.20.Ls, 78.70.Ck

Keywords: magnetic films, exchange-coupling, magneto-optical effects, x-ray spectroscopy

Corresponding Author: J. B. Kortright, Lawrence Berkeley National Laboratory, 1 Cyclotron Road, Berkeley, California, 94720 USA. (510) 486-5960 phone, (510) 486-5530 fax, JBKortright@lbl.gov. 


\section{Introduction}

The soft x-ray range contains relevant core levels of most species that constitute magnetic materials, including the $3 d$ transition elements and many rare earth elements. Relevant core levels are those from which dipole allowed transitions occur to final states containing spin-polarized holes, resulting in large, resonant magneto-optical (MO) effects. A comprehensive overview of soft x-ray methods to study magnetic materials properties of current interest was recently published [1]. Magnetic circular dichroism (MCD) was the first MO effect measured from ferromagnets in the soft x-ray range [2,3], in part because of the relative ease of measuring absorption using electron yield techniques and elliptical polarization. A variety of "photon-out" MO effects can be classified as Faraday effects in transmission or Kerr effects in reflection, and have advantages over electron yield techniques including deeper penetrating power and applicability in strong and varying applied field $\mathbf{H}$ to measure reversal behavior. The utilization of photon-based MO effects is still emerging in the soft x-ray range, in part because they manifest as polarization as well as intensity changes on reversal of sample magnetization $\mathbf{M}$, necessitating development of tunable linear polarizers $[4,5]$ to measure them completely. Other complications include strong interference in layered thin films and the need for reliable values of resonant magneto-optical constants and appropriate theoretical models [6].

In this paper we present early measurements of magnetization reversal in novel exchange-spring films. These heteromagnetic materials are of interest as a means to increase the maximum stored energy product beyond values obtainable from single phase magnets [7]. Such structures utilize strong interfacial exchange between hard and soft magnetic layers to yield interesting 3-dimensional (3-d) spiral or twist magnetization structure on reversal $[8,9]$. The long term goal of these measurements is a detailed experimental understanding of the 3-d reversal behavior of the hard and soft layers and comparison with theoretical models in an effort to tailor the properties of these nanocomposite magnetic systems. These early measurements are aimed at testing the sensitivity of various x-ray MO Kerr effect (XMOKE) techniques (both experimental and analytical) to the complex magnetization structure that exists in the soft layer in applied fields resulting from its exchange coupling to the hard layer. The spectral 
dependence of certain XMOKE signals is studied at fixed incidence angle, and Kerr intensity and rotation hysteresis loops measured using linear and/or elliptical polarization are compared. Sensitivity to transverse as well as longitudinal moments present in the twist structure requires measurement of both Kerr rotation and intensity signals, and those measured with linear polarization provide the most simply interpreted signals. With slight variations in energy near the Fe $L_{3}$ line the depth sensitivity in the reversal of the soft Fe layer is significantly altered, allowing reversal of either just the top of the Fe layer or the entire Fe layer to be sensed. Results demonstrate qualitative agreement of measured and calculated signals, and the potential for quantitative modeling of XMOKE measurements from well characterized samples with realistic knowledge of resonant MO properties.

\section{Experiment}

An endstation designed for XMOKE experiments was used for measurements reported here. Important features of this instrument are shown schematically in Fig. 1. Kerr or Faraday geometry samples are positioned between the poles of an electromagnet producing a maximum field greater than $1.5 \mathrm{~T}$ in the 1 inch gap. Incident and scattered x-rays transit through openings in the poles to detectors downstream of the electromagnet. One of two different detectors can be used at a given time. The downstream detector is a tunable multilayer linear polarizer $[4,5]$ used to measure polarization and polarization changes. A laterally graded $\left[\mathrm{W} / \mathrm{B}_{4} \mathrm{C}\right]_{200}$ multilayer with period range designed to span the $\mathrm{Fe}$ $L_{2,3}$ edge was used in these experiments. For XMOKE rotation measurements the azimuth of the polarizer was set at $45^{\circ}$ with respect to the incident linear polarization. Upstream of the polarization detector an intensity detector can be translated into the beam to measure Kerr intensity changes accompanying MO effects. This endstation enables a variety of resonant soft x-ray MO measurements in which field, scattering vector (or incident angle), photon energy, and sample temperature are varied.

Results reported here were obtained with this endstation positioned in the beam from the elliptically polarizing undulator (EPU beamline 4.0) at the Advanced Light Source at LBNL. Prior to MO measurements, standard rotating analyzer techniques were used to measure directly the degree of linear polarization $P_{L}$ of the beam radiated from the source at the energy of the Fe $L_{3}$ edge. Linear horizontal 
and vertical polarization were measured to have $P_{L} \geq 0.99$. At a different EPU tune the degree of circular polarization $P_{C}$ was indirectly determined as $P_{C}=\sqrt{1-P_{L}}=0.90$ where $P_{L}$ is directly measured and we assumea negligible amount of unpolarized radiation.

The sample studied here is one of a set studied by other techniques in early investigations of exchange-spring behavior [8]. It consists of quasi-epitaxial layers grown at elevated temperatures on a $\mathrm{MgO}$ (110) substrate having nominal structure $\mathrm{MgO} / \mathrm{Cr}(20 \mathrm{~nm}) / \mathrm{Sm}-\mathrm{Co}(20 \mathrm{~nm}) / \mathrm{Fe}(20 \mathrm{~nm}) / \mathrm{Cr}(5 \mathrm{~nm})$. It has strong in-plane uniaxial anisotropy, and measurements were made along the easy axis. In weak and moderate reverse fields, the top of the Fe layer reverses while the bottom of the Fe layer remains pinned at the interface with the high anisotropy Sm-Co layer forming a twist or spiral in depth of the Fe magnetization analogous to a Bloch domain wall in the plane of the film. The twist structure is entirely reversible in reverse fields below the coercive field of the Sm-Co layer (reduced by exchange coupling with $\mathrm{Fe}$ ), at which point the hard layer and hence entire structure switch irreversibly. A one dimensional model describing $\mathbf{M}$ vs depth and $\mathbf{H}$ in the hard and soft layers was developed that characterizes the magnetization dynamics of the entire structure [8]. This model subdivides the Fe and Sm-Co layers into finer layers parallel to the film plane and calculates the longitudinal and transverse moments within each sublayer from an iterative model of exchange coupling. This one-dimensional model assumes coherent rotation of magnetization at a given depth in the structure, and yields a twist or spiral magnetization structure whose characteristics in depth depend on $\mathbf{H}$.

This model also provides fundamental information on the in-plane orientation of $\mathbf{M}$ with depth required to calculate the Kerr rotation and intensity signals for comparison with measured signals. We use the MO formalism of Zak, et al., [10], that allows for arbitrary orientation of $\mathbf{M}$ in arbitrarily many layers describing the sample. This formalism provides for evaluation of the Kerr matrix of reflected amplitudes $\left(\begin{array}{cc}r_{s s} & r_{s p} \\ r_{p s} & r_{p p}\end{array}\right)$ from which the complex Kerr rotation is obtained as $\phi_{K}=r_{p s} / r_{s s}=\alpha_{K}+i \varepsilon_{K}$ for incident s polarization, where $\alpha_{K}$ and $\varepsilon_{K}$ are the Kerr rotation angle and ellipticity angle, respectively. 
The Kerr intensity is simply the total intensity represented by all four amplitudes for a given incident polarization. Interface and propagation matrices are defined for each layer of different chemical composition, and the $20 \mathrm{~nm}$ Fe layer is subdivided into layers of $0.21 \mathrm{~nm}$ each as in the magnetization model describing the twist structure in the Fe layer. Resonant, helicity-dependent magneto-optical constants measured from a $32 \mathrm{~nm}$ Fe film not coupled to other magnet layers were used to describe the Fe layer [6], and tabulated values for the $\mathrm{Cr}$ and $\mathrm{Sm}$-Co layers and the $\mathrm{MgO}$ substrate [11]. In the optical model the $\mathrm{Cr}$ capping layer is assumed to have oxidized to form $\mathrm{Cr}_{2} \mathrm{O}_{3}$, consistent with $\mathrm{Cr}$ absorption spectra from similar thin capping layers.

\section{Results \& Discussion}

Thin film interference effects are known to impact the scattering vector, and hence spectral, dependence of x-ray MO signals [6]. Thus it is useful to investigate this spectral dependence both to understand and possibly model the interference effects and to select an energy where MO signals are large and well away from zero crossings where the sign of MO signals change. Fig. 2a shows the measured asymmetry ratios for Kerr rotation measured using incident linear (s-component) polarization and Kerr intensity measured using incident elliptical polarization $\left(P_{C}=0.90\right)$. These data and all data below were collected at a fixed grazing incident angle of $\theta=3.6^{\circ}$, which is just above the critical angle for total external reflection for the capping layer to enable significant penetration into the soft Fe layer of interest. The asymmetry ratios are obtained from the difference over the sum of spectral scans taken with the sample saturated in opposite directions. This definition is necessary in evaluating the spectral shape of the Kerr rotation signal, which (for efficiency) was measured with the tunable multilayer polarizer at a fixed energy tune position. Thus the individual spectral scans at opposite saturation show Kerr rotation features superimposed with features of the spectral reflectivity across the multilayer Bragg peak (and the sample's Kerr intensity). In taking the normalized asymmetry ratio the spectral dependence of the multilayer is removed, leaving a signal that is proportional to the Kerr rotation. The asymmetry ratio of the Kerr rotation shows a sharp peak at $704.7 \mathrm{eV}, 2.3 \mathrm{eV}$ below the peak of the Fe $L_{3}$ white line at 707 $\mathrm{eV}$, and a broader oscillation. The asymmetry ratio of the Kerr intensity using elliptical polarization 
shows similar features that are systematically shifted in energy with respect to those of the Kerr rotation signal using linear polarization.

Calculated asymmetry ratios for the same quantities are in Fig. 2b, and show similar spectral features to the measured quantities in Fig. 2a. Calculations assume the above nominal structure for this sample. They reveal strong sensitivity of the shape of asymmetry spectra to the thickness of the individual layers in the sample, to the incidence angle, and to the optical properties especially of Fe that are undergoing strong resonant changes across this region. The broad oscillation is due to interference effects, while the sharp feature at $704.7 \mathrm{eV}$ is a resonant optical effect. The general agreement in shape of calculated and measured spectra, especially the sharp feature, imply that the magneto-optical model provides a reasonable approximate description of the interference and resonant magneto-optical effects present in the sample. No attempt has been made to adjust parameters to improve the agreement of calculated and measured spectra, and it is possible that systematic attempts to do so will result in improved agreement. The calculations indicate that the asymmetry ratio of the Kerr intensity measured with circular polarization closely approximates the Kerr ellipticity. Thus the two different curves in both panels of Fig. 2 are approximately related via a Kramers-Kronig transformation, as is consistent with their general appearance. The energy of $704.7 \mathrm{eV}$ was selected for Kerr rotation and intensity hysteresis measurements, since this energy yields the largest rotation.

XMOKE hysteresis loops measured using linear polarization are shown in Fig. 3. The raw Kerr rotation signal is in Fig. 3a, and is the signal directly measured in the detector downstream of the linear polarizer. This signal exhibits a clear asymmetry under reversal initiated from states of opposite saturation. The Kerr intensity signal, measured upstream of the polarizer, is in Fig. 3b, and is symmetric under reversal from states of opposite saturation. This Kerr intensity signal also modulates any Kerr rotation signal, and the true Kerr rotation signal is obtained by normalizing the raw signal in Fig. 3a by the intensity signal in Fig. 3b. The normalized result is then converted to Kerr rotation angle in Fig. 3c knowing the azimuth of the polarizer in the beam reflected from the sample. The size of the measured Kerr angle is much larger than that typically observed in near-visible spectral regions, consistent with the 
large size of resonant MO signals at core resonances [5,6]. The normalized Kerr angle is free from the asymmetry in the raw signal.

Qualitative interpretation of the XMOKE loops results from knowing that, in the longitudinal geometry used here, Kerr rotation signals result from changes in the longitudinal moment, while Kerr intensity signals for linear polarization are proportional to changes in net transverse moment through the hysteresis loop [12]. The shape of the Kerr angle loop reveals the reversal of part of the Fe layer at low reverse field $(\sim 0.09 \mathrm{~T})$, and a more gradual reversal of other parts of the Fe layer extending to roughly $0.75 \mathrm{~T}$ at which point the entire Fe layer reverses. The distinctly different shape of the Kerr intensity loop shows abrupt changes at the same reverse fields as those in the Kerr rotation data, and similar gradual change between these abrupt changes. These shapes are qualitatively consistent with the expected reversal behavior of the Fe layer along the easy axis. At roughly $0.09 \mathrm{~T}$ the top of the Fe layer quickly reverses with increasing $\mathbf{H}$, yielding the rapid drop in Kerr angle and increase in Kerr intensity as the magnetization spiral in the Fe layer is introduced which necessarily produces a net transverse moment accounting for the intensity signal. With increasing reverse field more of the Fe layer reverses from the top and the domain wall is pushed downward toward the Sm-Co layer. The Sm-Co layer yields to reversal at $0.75 \mathrm{~T}$ along with the bottom of the Fe layer that is strongly influenced by exchange coupling to it.

It is instructive to compare the shapes of XMOKE signals measured using linear incident polarization with those measured using near-circular polarization. This is because most early measurements of resonant XMOKE spectra and hysteresis loops have used elliptical polarization to measure Kerr intensity changes, generally without the ability to measure phase or polarization changes. Thus we measured the sample under the same conditions $(h v, \theta)$ except using elliptical polarization as noted above. The results for the same signals are in Fig. 4. Neither the raw Kerr rotation loop (Fig. 4a) nor the Kerr intensity loop (Fig. 4b) are symmetric on reversal from opposite saturation. However the normalized Kerr angle (Fig. 4c) is symmetric with shape identical to that measured using linear polarization. 
The differences and similarities of loops measured with elliptical and linear polarization are understood by realizing that linear polarization can be described as equal amounts of opposite helicity circular components, while elliptical polarization can be described as unequal amounts of opposite helicity circular components or, alternatively, as having both circular and linear character. Since opposite helicity circular components generally have different reflected intensities even from an Fe film with $\mathbf{M}$ saturated in the longitudinal direction [6], the saturation Kerr intensities are expected to be different for opposite saturation with elliptical polarization but identical for linear polarization. This general asymmetry in Kerr intensities for elliptical polarization persists in the field region where the twist structure exists, while the general symmetry in Kerr intensity remains for incident linear polarization. These general trends are supported by MO calculations described above and in ref. 6 . The observed raw Kerr rotation has an asymmetry different from the Kerr intensity, indicating that a real rotation signal exists in the data resulting largely from the partial linear character of the incident elliptical polarization and revealed as the normalized Kerr angle in Fig. 4c. The magnitude of the Kerr angle measured using elliptical polarization is significantly smaller than that measured using linear polarization, consistent with the reduced linear character of the elliptical polarization.

This inherent asymmetry of Kerr intensity signals to $\mathbf{M}$ reversal for incident elliptical polarization generally complicates their interpretation in terms of longitudinal $\mathbf{M}$ components, and especially in terms of transverse components present in the reversal of this exchange spring film. Thus hysteresis loops measured only with Kerr intensity and elliptical or circular polarization can be expected to exhibit unusual shapes. Realistic calculations of XMOKE intensities, i.e., using full MO formalisms including interference, measured optical constants for actual structures, and accurate incident polarization, may aid in interpreting loops measured using elliptical polarization in terms of transverse and longitudinal moments. However, the two separate measurements of Kerr rotation and intensity using linear polarization offer the most direct access to longitudinal and transverse moment determination in the spring sample studied here. It should be noted that a Kerr intensity signal is often absent in hysteresis 
loops of in-plane films, in which case it's absence is taken as an indication that there is no net transverse moment during the reversal process.

The Kerr angle loops measured with both linear and elliptical polarization are plotted together with a calculated Kerr angle loop in Fig. 5. The shapes of the measured loops are in good agreement, indicating that they have similar sensitivity to changes in longitudinal moments. The calculation is made using the multilayer MO formalism as described above. Each of the Fe sublayers has in-plane $\mathbf{M}$ of fixed magnitude but with longitudinal and transverse components that vary systematically in depth as determined in ref. 8 at discrete $\mathbf{H}$ values corresponding to the points in Fig. 5. The calculations in ref. 8 used anisotropy constants appropriate for low temperatures, and hence predict a much higher switching point for the hard Sm-Co layer than is observed in our room temperature measurements. The room temperature twist structure in the Fe layer is expected to be reasonably well described by this model. There is qualitative agreement in the shapes of measured and calculated Kerr angle loops, each showing the sharp low field switching of the top of the Fe layer followed by a gradual reversal of the deeper Fe sublayers as the domain wall is pushed toward and into the Sm-Co layer with increasing field. The calculated and measured (linear polarization) Kerr intensity loops also show general qualitative agreement in shape. At this early stage of development we do not find quantitative agreement measured and calculated XMOKE results, both spectral and field dependent. The lack of quantitative agreement could result from many factors, including imprecise structural knowledge of the actual layered sample, imperfect optical and/or magneto-optical constants describing the data, or the failure of the sample to respond as the idealized 1-dimensional magnetization model predicts. Further work will investigate these and other possibilities.

The qualitative consistency of measured and calculated loop shapes in Fig. 5 is an indirect, partial confirmation of the depth-dependence of the direction of $\mathbf{M}$ in Fe sublayers with the predicted exchangespring twist structure. More direct measures of the actual depth dependence of Fe magnetization in the sample can be obtained through different approaches. One approach is to tune photon energy near the Fe $L_{3}$ edge, since Fe absorption and hence penetration depth vary strongly at the $L_{3}$ white line. Another 
approach is to vary $\theta$ to change the electric field intensity profile in depth into the sample. A third approach is to grow chemically distinct magnetic marker layers into samples at different depths [9]. The first approach is demonstrated here, using Kerr intensity measurements made with elliptical polarization at the same $\theta=3.6^{\circ}$ but with photon energy tuned to $708 \mathrm{eV}$, corresponding roughly to the maximum in the measured Kerr asymmetry ratio in Fig. 2 and $1 \mathrm{eV}$ above the Fe $L_{3}$ line peak. The Kerr intensity loop measured under these conditions is shown in Fig. 6 to have a high degree of symmetry on reversal from opposite saturated states, with almost all motion of Fe magnetization completed below $0.25 \mathrm{~T}$. At this energy the (polarization averaged) penetration depth in Fe is expected to be more than an order of magnitude smaller than at $704.7 \mathrm{eV}$ used to measure loops in Figs. 3-5 [6]. Thus it is concluded from experiment alone that the loop in Fig. 6 represents reversal of just the top of the $20 \mathrm{~nm}$ thick Fe layer. Furthermore, the gradual changes in XMOKE loops out to $0.75 \mathrm{~T}$ at $704.7 \mathrm{eV}$ do result from motion of $\mathrm{Fe}$ magnetization in deeper regions of the Fe layer. The very different shapes of Kerr intensity loops measured with elliptical polarization in Figs. $4 \mathrm{~b}$ and 6 further emphasize the need for care in using this measure to infer magnetization dynamics in complex samples.

\section{Conclusions}

The 3-dimensional magnetization reversal behavior of novel exchange-spring films was used to test the sensitivity of various XMOKE measurements using different incident polarization and photon energy. These structures, and possibly other strongly exchange-coupled films, exhibit a net transverse moment on reversal and also a depth-dependent moment, requiring experimental techniques that can resolve these important characteristics. Resonant Kerr rotation and intensity measurements together provide direct sensitivity to longitudinal and net transverse moment changes, and measurements made using incident linear polarization are more directly interpreted (by their increased symmetry) than are measurements using elliptical or circular polarization. Kerr intensity loops alone measured using elliptical polarization are influenced by both longitudinal and transverse moment changes, but do not directly resolve them from each other. Working well below the $\mathrm{Fe} L_{3}$ line minimizes absorption and 
provides sensitivity to deeply buried layers, while tuning to and above the $L_{3}$ line enhances sensitivity to the top surface, providing a clear advantage compared to visible MOKE measurements that lack such sharp, strong spectral resonances. Strong interference effects are generally present in layered samples, demonstrating the need for theoretical models incorporating interference in interpreting data. Application of magneto-optical formalisms common in near-visible spectral regions together with measured resonant magneto-optical constants for Fe were shown to give qualitative agreement with measured MO spectral and field-dependent signals. These results highlight several features of resonant XMOKE techniques that are beyond the capabilities of near-visible MOKE and other common techniques for studying magnetization structure and dynamics nanostructured, multicomponent magnetic systems.

\section{Acknowledgements}

We are grateful to A.T. Young and E. Arenholz for their assistance in operating EPU beamline 4 at the ALS. Work at LBNL and ANL was supported by the Director, Office of Science, Office of Basic Energy Sciences, Division of Materials Science of the U.S. Dept. of Energy under contracts No. DE-AC0376SF00098 and W-31-109-ENG-38, respectively.

\section{References}

${ }^{*}$ Current address: Department of Physics and Center for Nanospinics of Spintronic Materials, Korea Advanced Institute of Science and Technology, Taejon 305-701, Korea.

1. J. B. Kortright, D. D. Awschalom, J. Stöhr, Y. U. Idzerda, S. S. P. Parkin, I. K. Shuller, and H.-C. Siegmann, J. Magn. Magn. Mater. 207, 7 (1999).

2. C. T. Chen, F. Sette, and S. Modesti, Phys. Rev B 42, 7262 (1990).

3. J. G. Tobin, G. D. Waddill, and D. P. Pappas, Phys. Rev. Lett. 68, 3642 (1992).

4. J. B. Kortright, M. Rice and K. D. Franck, Rev. Sci. Instrum. 66, 1567 (1995).

5. J. B. Kortright, M. Rice and R. Carr, Phys. Rev. B 51, 10240 (1995).

6. J. B. Kortright and Sang-Koog Kim, Phys. Rev. B 62, 12216 (2000).

7. E. F. Kneller and R. Hawig, IEEE Trans. Mag. 27, 3588 (1991).

8. E. E. Fullerton, J. S. Jiang, M. Grimsditch, H. C. Sowers, and S. D. Bader, Phys. Rev. B 58, 12193 (1998).

9. O. Hellwig, J. B. Kortright, K. Takano, and E. E. Fullerton, Phys. Rev. B 62, 11694 (2000). 
10. J. Zak, E. R. Moog, C. Liu, and S. D. Bader, Phys. Rev B 43, 6423 (1991), and errata ibid, 46, 5883 (1992).

11. B. L. Henke, E. M. Gullikson, and J. C. Davis, Atomic Data and Nuclear Data Tables 54, 181 (1993). Numerical data (in some cases updated since this publication) can be found at http://wwwcxro.lbl.gov/optical_constants/.

12. M. J. Frieser, IEEE Trans. Mag. 4, 152 (1968). 


\section{Figure Captions}

Figure 1. Schematic of apparatus used for x-ray magneto-optical Kerr effect (XMOKE) measurements showing the sample between the poles of an electromagnet. Incident and scattered x-ray beams travel through openings in the poles to intensity and polarization detectors downstream of the magnet. Grazing incidence angles from $0^{\circ}$ to $14^{\circ}$ are possible, enabling a variety of Kerr (reflection) and Faraday (transmission) geometry experiments.

Figure 2. Measured (a) and calculated (b) spectral asymmetry ratios of XMOKE signals corresponding to Kerr rotation measured using linear polarization (solid line) and Kerr intensity measured using elliptical polarization (dashed line) in the vicinity of the Fe $L_{3}$ edge (at $707 \mathrm{eV}$ ). Each asymmetry ratio is determined as the difference over the sum of spectral scans measured with the exchange spring sample saturated along the easy axis in a longitudinal geometry. Lines connect measured and calculated data points. Structure in the asymmetry ratios results from both interference effects in the layered sample together with strong resonant $\mathrm{MO}$ properties of Fe near it $2 p$ resonances.

Figure 3. XMOKE hysteresis loops measured using linear (s) polarization at fixed incidence angle and $h v=704.7 \mathrm{eV}$. The raw Kerr rotation signal is in (a), and the Kerr intensity signal measured before the polarizer is in (b). In (c) is the Kerr rotation angle obtained by first normalizing the raw Kerr rotation signal by the intensity signal and converting the result to rotation angle. Lines connect measured and normalized data points.

Figure 4. XMOKE hysteresis loops measured using linear (s) polarization at fixed incidence angle and $h v=704.7 \mathrm{eV}$. The raw Kerr rotation signal is in (a), and the Kerr intensity signal measured before the polarizer is in (b). In (c) is the Kerr rotation angle obtained by first normalizing the raw Kerr rotation 
signal by the intensity signal and converting the result to rotation angle. Lines connect measured and normalized data points.

Figure 5. Kerr angle loops obtained with linear and elliptical polarization are plotted together with a calculated Kerr angle loop. All loops are normalized to saturation values. Lines connect measured and calculated data points.

Figure 6. Kerr intensity loop measured roughly $1 \mathrm{eV}$ above the $\mathrm{Fe} L_{3}$ line using elliptical polarization. At this energy penetration of the electric field into the Fe layer is severely limited, confirming that it is the top of the Fe layer that reverses at low applied fields. Lines connect data points. 


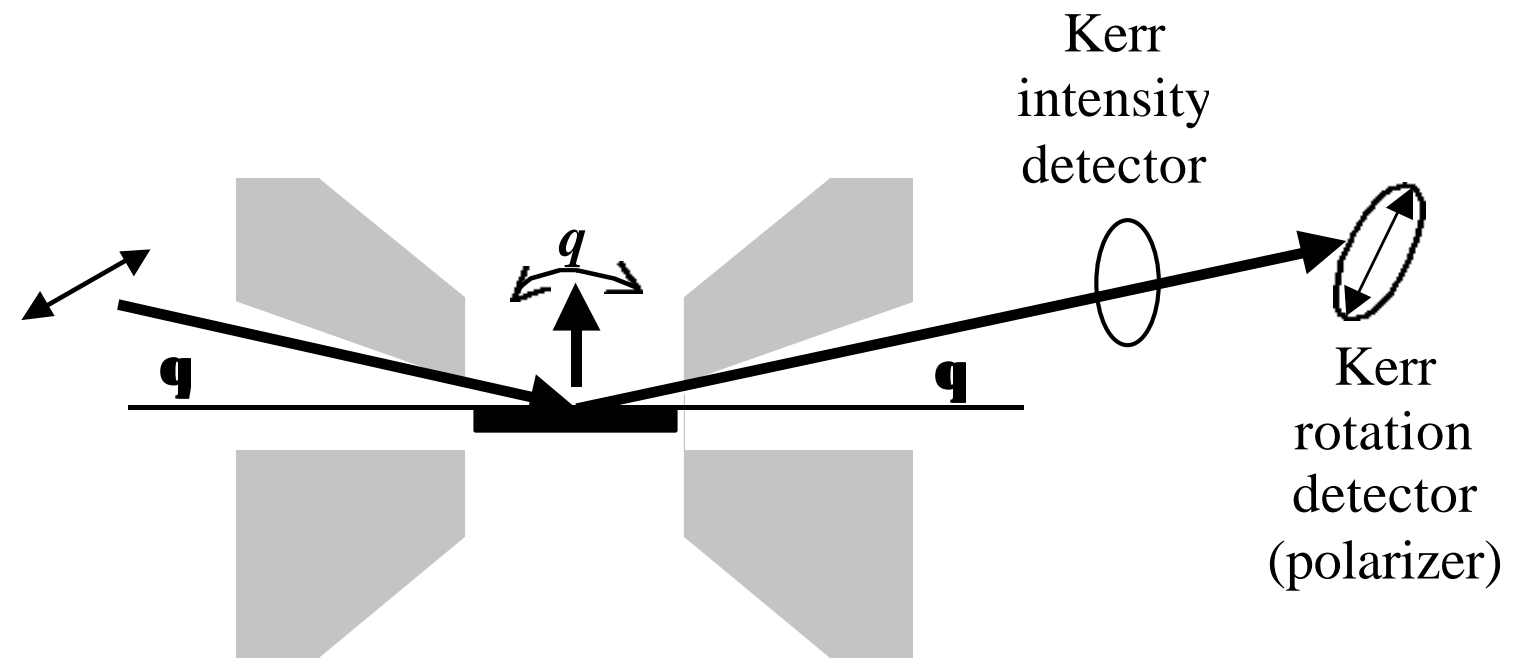

Figure 1 

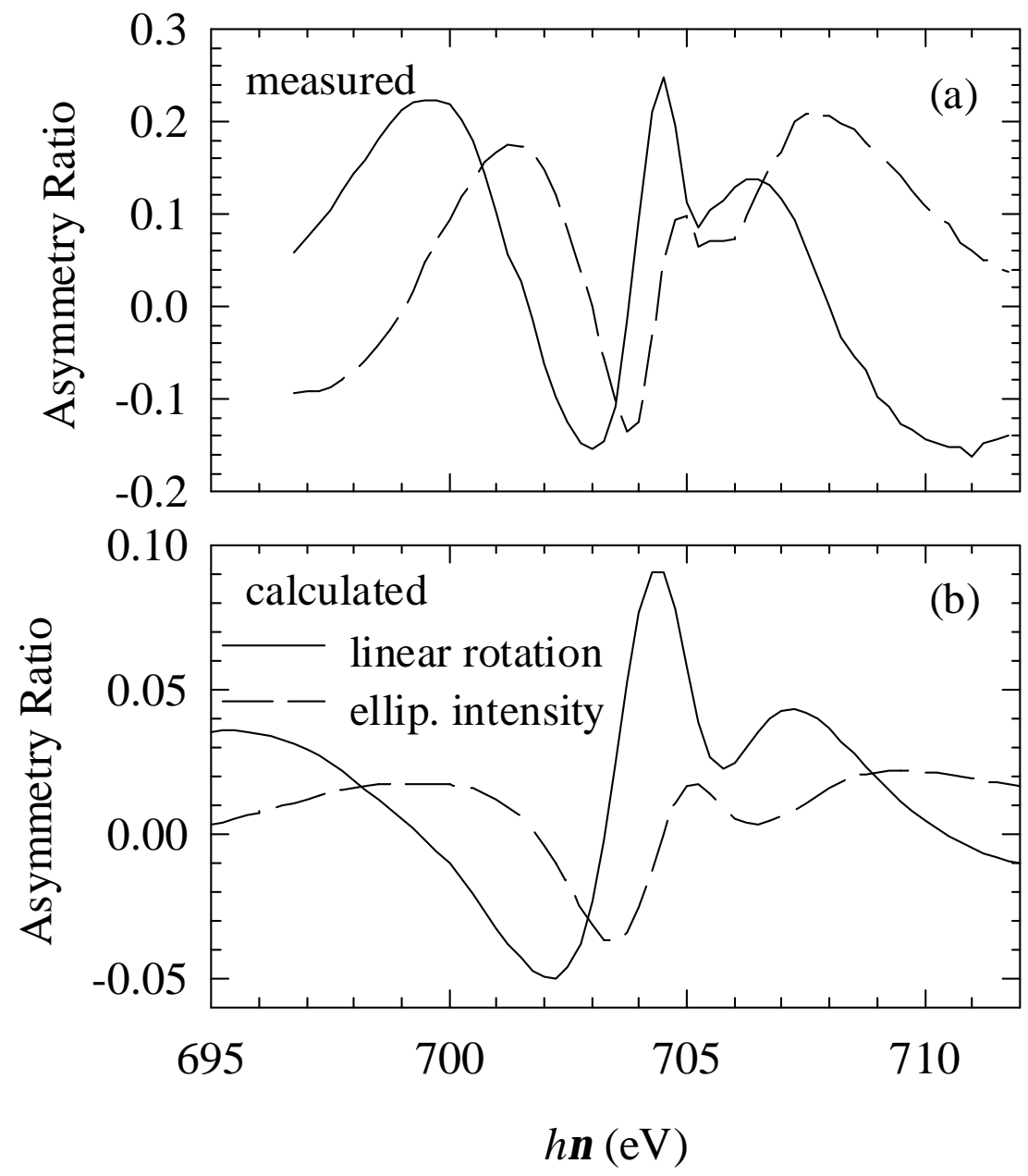

Figure 2 

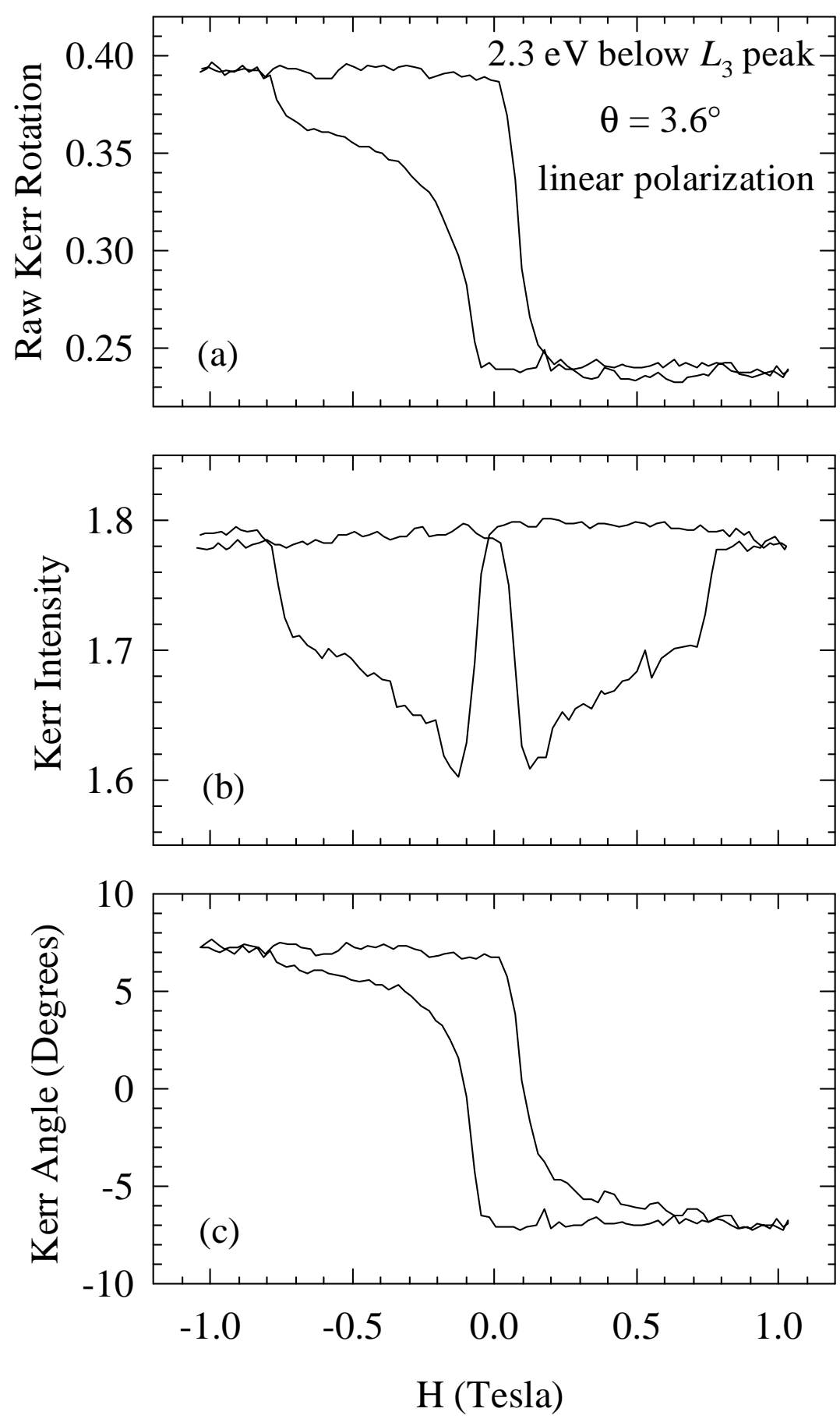

Figure 3 

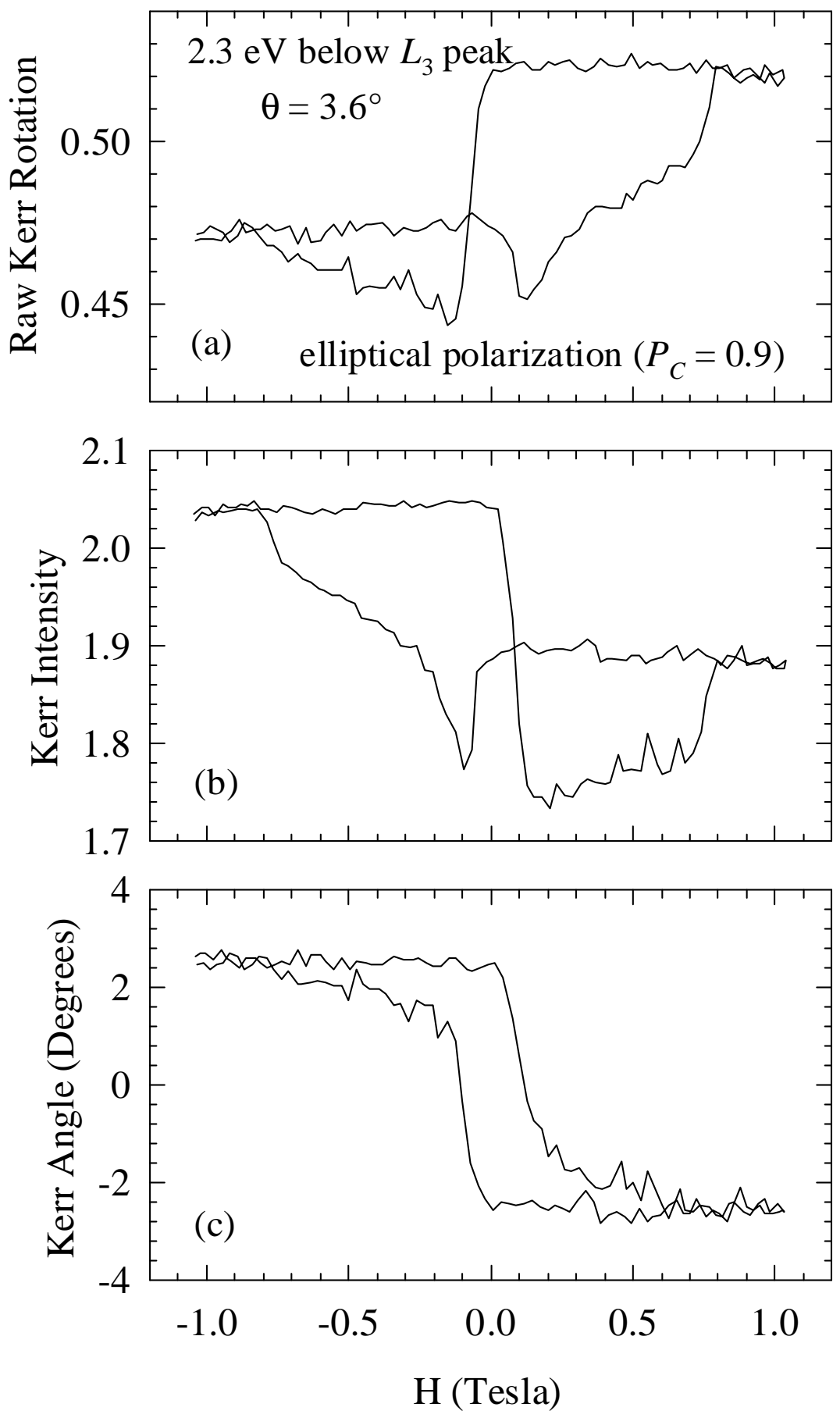

Figure 4 


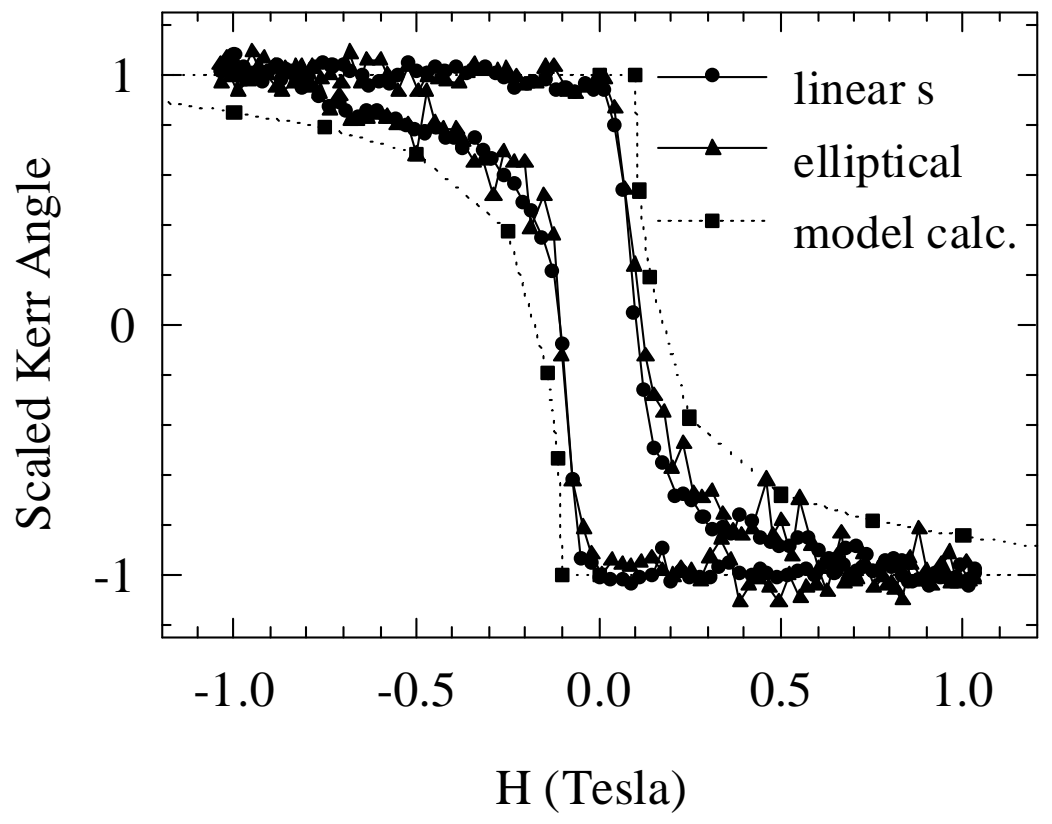

Figure 5 


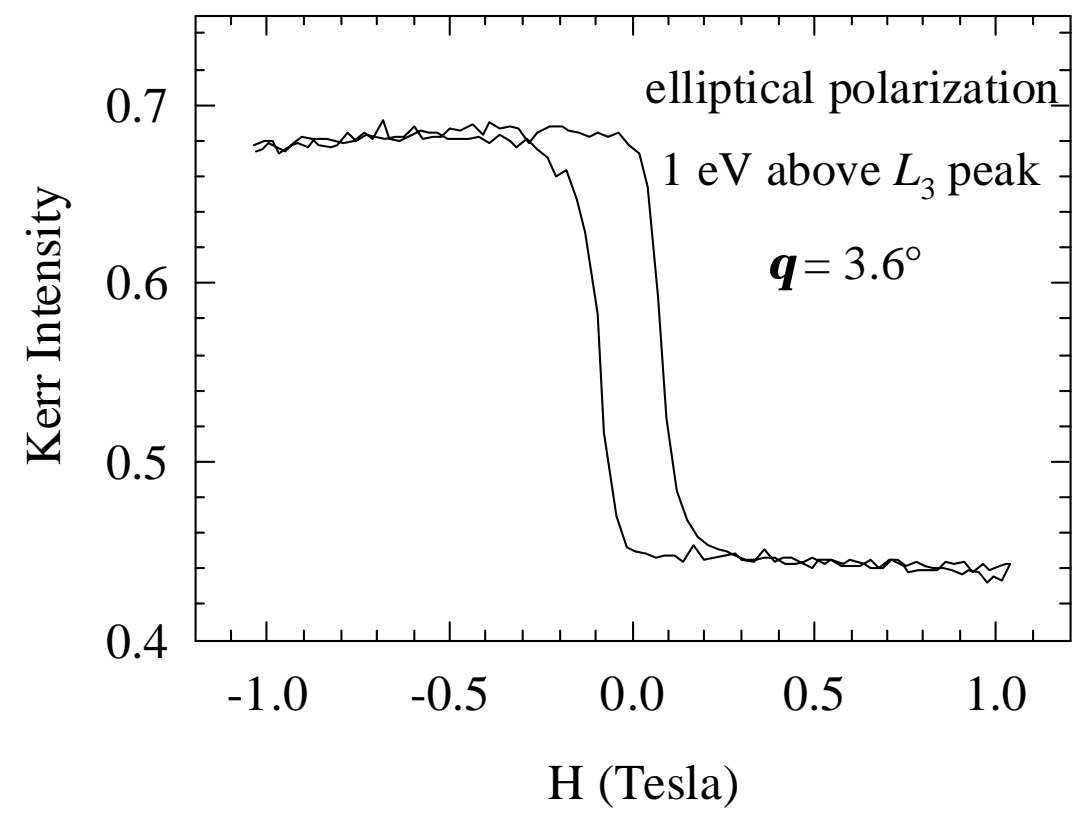

Figure 6 